\title{
A Clinical Case Study of Paratesticular Rhabdomyosarcoma of Young Adult
}

\section{Bai $W^{\star 1}$, Chadli A ${ }^{1}$, Moataz A ${ }^{1}$, Dakir $\mathrm{M}^{1}$, Debbegh A ${ }^{1}$, Aboutaieb R ${ }^{1}$, Taoussi R ${ }^{2}$, Qechchar $\mathrm{Z}^{2}$, Tabakh $\mathrm{H}^{2}$,} Touil $\mathrm{N}^{2}$, Kacimi $\mathrm{O}^{2}$ and Chikhaoui $\mathrm{N}^{2}$

${ }^{1}$ Department of Urology, Ibn Rochd de Casablanca, Morocco

${ }^{2}$ Surgical Emergency Radiology Department, IBN ROCHD CHU

*Corresponding author: Bai W, Department of Urology, Ibn Rochd de Casablanca, Morocco, Tel: 00212662336904, E-mail: walid2724@hotmail.fr

Citation: Bai W, Chadli A, Moataz A, Dakir M, Debbegh A, et al. (2019) A Clinical Case Study of Paratesticular Rhabdomyosarcoma of Young Adult. J Case Rep Stud 7(1): 109. doi: 10.15744/23489820.7.109

Received Date: August 16, 2018 Accepted Date: February 26, 2019 Published Date: February 28, 2019

\begin{abstract}
Para-testicular rhabdomyosarcoma (RPT) is a rare tumor of children and adolescents. The diagnosis is made by the anatomopathological study. The treatment must be multimodal and involves surgery, chemotherapy and radiotherapy. We report the case of a 19-year-old adolescent in our department for an embryonic-type para-testicular rhabdomyosarcoma. Our aim is to expose a rare and unknown case that may be life-threatening. We would like to emphasize on the fact that paratesticular rhabdomyosarcoma need to be brought up in front of a tissular process expanding in the scrotum with normal testicules.
\end{abstract}

Keywords: Rhabdomyosarcoma; Paratesticular tumor; Exploration; Prognosis; Treatment

\section{Introduction}

Rhabdomyosarcoma (RMS) is a rare mesenchymal tumor characterized by the presence of cells identifiable with rhabdomyoblasts. The genitourinary sites of sarcomas are among the most frequent (34\%) [1]. Paratesticular localization accounts for only $7 \%$ of intrascrotal neoplastic sites [2,3]. The embryonic variant is the most common [4]. Furthermoren, differentials often arise with scrotal emergencies that may hide the malignant nature of the pathology. These tumors are characterized by their rapid evolution and poor prognosis if diagnosed lately [5]. The treatment must be multimodal and involves surgery, chemotherapy and radiotherapy. We report the case of a 19-year-old adolescent in our department for an embryonic-type para-testicular rhabdomyosarcoma. Our goal is to expose a rare and unknown case that may be life-threatening.

\section{Observation}

Our case was one of a 19 years old male, with no particular history, consults for a large painless purse evolving for about 3 months.

The clinical examination found an afebrile patient in good general condition, the two purses increased in volume of regular contours, with a mass that seems independent of the testicles, of hard consistency, painless on palpation and without local inflammatory signs (Figure 1). The trans-illumination is negative and the rest of the clinical examination is normal with no signs of lymphadenopathies.

The ultrasound performed at admission shows a voluminous medial intrascrotal tissular mass of heterogeneous structure, echoic, containing small areas of necrosis, with small vascularity especially at the periphery, it measures approximately 12.5 by 8.5 by 8.0 centimeters, repressing the two testicles at the top and laterally which are of normal size and echo structure without obvious nodular lesion seen.

Tumor marker assay $(\mathrm{HCG}=1 \mathrm{ng} / \mathrm{ml}, \mathrm{LDH}=220 \mathrm{UI} / \mathrm{l}, \mathrm{AFP}=20 \mathrm{ng} / \mathrm{ml}$, ) were normal.

The patient underwent a pelvic MRI which revealed a heterogeneous tissular scrotal mass infiltrating the scrotal skin, without invasion of the testicles. This scrotal mass is developing in the perineum in contact with the dorsal surface of the penis and the anterior margin of the anal margin, with inguinal lymphadenopathy, the largest of which measures 19 by $13 \mathrm{~mm}$ (Figure 2 yellow arrow).

An ultrasound guided biopsy of the mass objected an embryonic type rhabdomyosarcoma.

The patient was presented at the multidisciplinary meeting of onco-urology, the decision was to make a first chemotherapy and then send it to us for complementary surgical excision. 


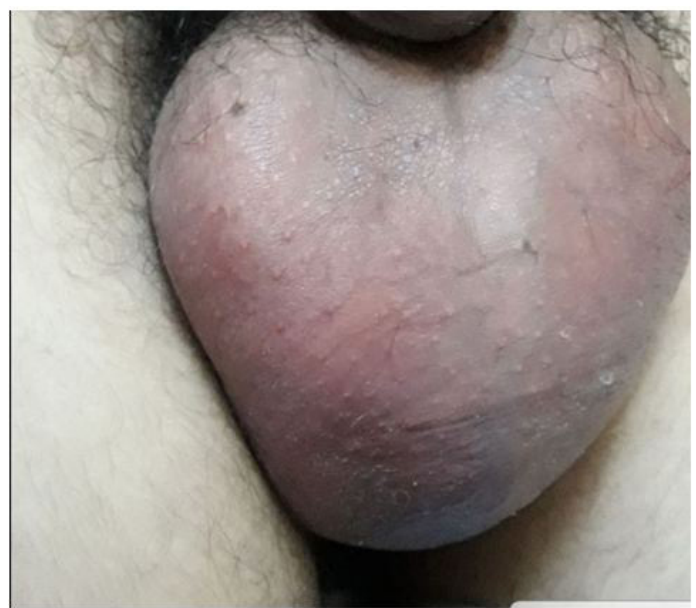

Figure 1: Clinical appearance showing an increased volume purse

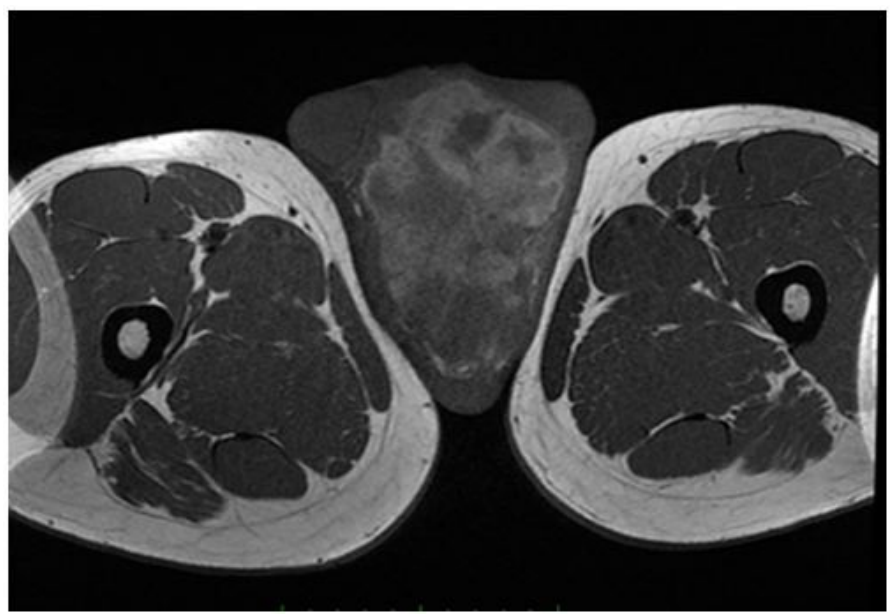

Figure 2: MRI T1 axial section

\section{Discussion}

Paratesticular RMS develops from the mesenchymal tissues of the spermatic cord, epididymis, and testicular tunicas. It represents $7 \%$ of all rhabdomyosarcomas $[6,7]$.

Paratesticular RMS is observed at any age, but especially in children and young adults. There are two peaks of incidence, one at age 4 and the other at age $16[8]$. There is no predilection of race.

Primitif paratesticular localization is considered to be of good prognosis compared to other rhabdomyosarcomas, despite the frequency of retroperitoneal lymph node involvement. Its superficial localization allows a rapid diagnosis and consequently an often-complete resection of the tumor.

Local spread is very early and distant spread is lymphatic and bloodstream [9-11]. The most frequent metastatic sites are retroperitoneal lymph nodes, lungs, liver and bones $[8,9,11]$. Tumor thrombosis of the inferior vena cava has also been described, requiring excision of the tumor and thrombus under extracorporeal circulation [12,13].

The clinical presentation is not unusual in comparision with other tumors with intraspecal development and the paratesticular localisation of the tumor is difficult to specify by physical examination only [11]. It is a hard mass, fast growing, rarely painful, which is seen to the left as to the right without predominance [8-11].

The determination of tumor markers (HCG, LDH, FP) must be part of the assessment of any testicular tumor, but it is often normal in paratesticular tumors. There are no tumor markers that can help approch the diagnosis, which is based only on the histological examination of the orchiectomy [9].

Scrotal ultrasound, first-line examination, despite its low specificity, will differentiate between testicular mass and extra testicular. We find a tissular mass at the expense of the scrotal soft tissues, extra-testicular, usually voluminous, hypoechoic or sometimes hyperechoic, of heterogeneous structure and with irregular margins [14]. This mass has an inguino-scrotal extension in $80 \%$ of cases. It may be associated with a hypoechoic appearance of the testicle and epididymis in relation to edema. Given its large volume, it may not always be attached to a specific structure or be distinguished from a testicular tumor. The color Doppler study will show a large vascularization of the mass that can delineate areas of necrosis in some cases [15]. 
MRI is the study of choice for the exploration of extratesticular masses. Its use will be of a great interest in their characterization as well as in case of significant locoregional extension to better appreciate the relationship of the tumor with the pelvic tissues [16]. It is carried out in sequences weighted in T1 and T2 with sometimes sequences in fat saturation. Although not necessary, gadolinium injection may be useful for the study of tumor vasculature. The tumor appears as an intermediate signal at T1 and T2, homogeneous at T1, and heterogeneous at T2. MRI is an excellent study for post-therapeutic monitoring by appreciating tumor residues or local recurrences. It also eliminates differential diagnoses such as lipoma, fibrous pseudotumors and polyorchidism [17] (Figure 2, 3, 4 and 5).

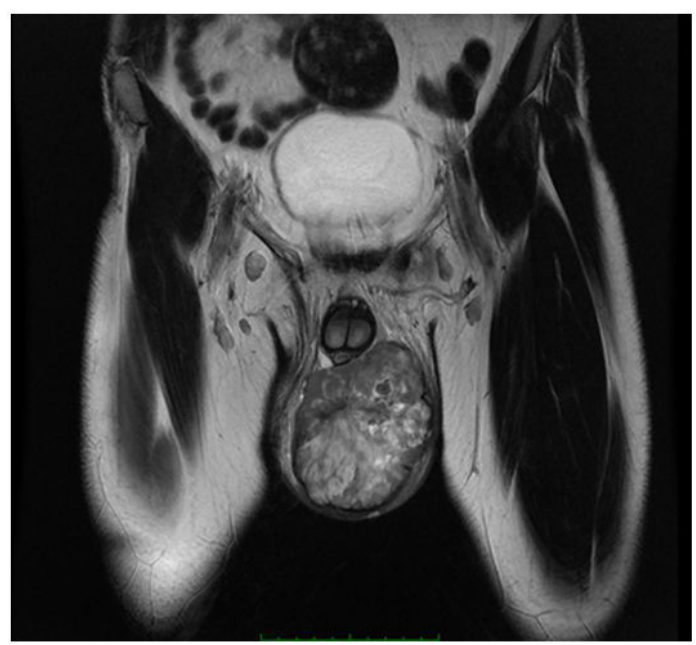

Figure 3: Coronal T2 MRI

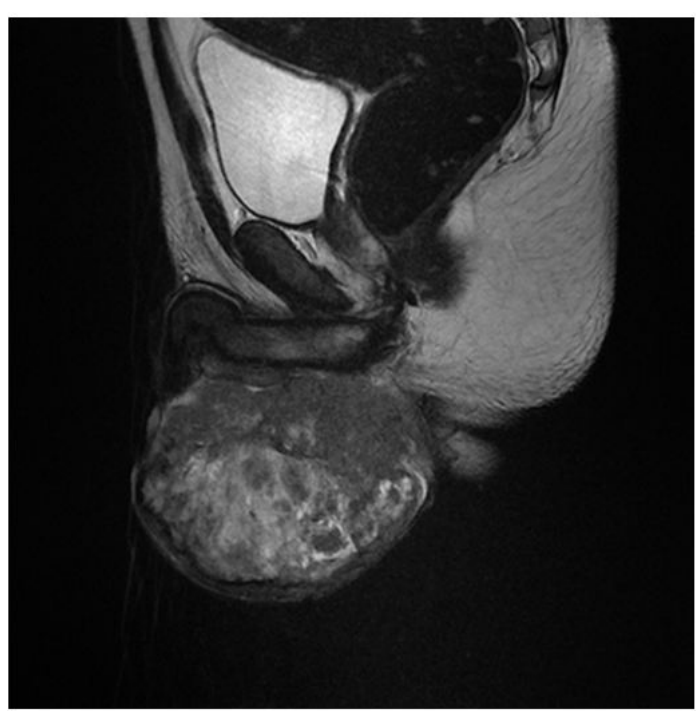

Figure 4: MRI T2 Sagittal section

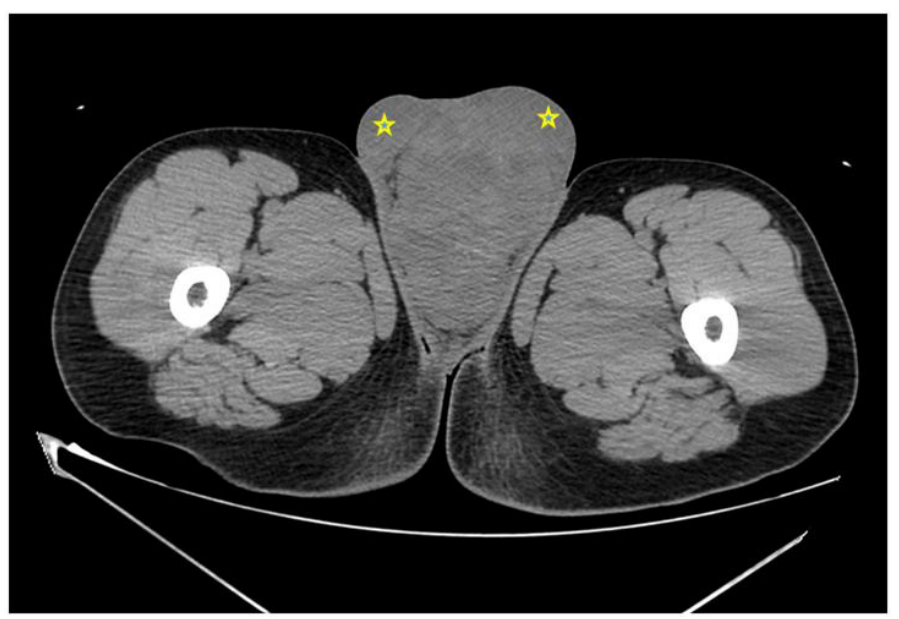

Figure 5: Axial CT scan showing a scrotal mass pushing back the testes (star) without invading them, isodense, heterogeneous delineating areas of necrosis and seat multiple scattered calcifications 
Currently, the CT is less indicated in the exploration of scrotal masses, because of irradiation and furthermore, brings less information as MRI does. The thoraco-abdominopelvic CT scan helps to assess the characteristics of the masses and its limits, to judge the distant extension of the disease as well as to explore the pelvic and lumbo-aortic lymph node areas, to search for hepatic, bone and / or pulmonary metastases. Before contrast, the paratesticular rhabdomyosarcoma appears isodense to the muscular structures, with or without calcifications. After injection of contrast, there is a heterogeneous enhancement with sometimes delimitation of hypodense areas of necrosis [18]

Extension assessment should be performed in search of hematogenous and lymphatic dissemination. More than $70 \%$ of rhabdomyosarcomas have retroperitoneal lymphadenopathies and 20\% distant metastases, most commonly in the lung and bone at the time of diagnosis. Abdominopelvic computed tomography (CT) allows the search for invasion of the deep ganglionic chains especially lumbar-aortic and pelvic and liver metastases [19].

The staging of tumors is based on the extension of the primary tumor and the state of the regional lymph nodes. The most commonly used classification is that proposed by the Intergroup Rhabdomyosarcoma Study (IRS) (Table 1).

\begin{tabular}{|l|}
\hline Stage 1: Localized tumor, resected in its entirety \\
\hline Stage 2A: Tumor resected with invasive margin of resection, without ganglia \\
\hline Stage 2B: Resected tumor with retroperitoneal lymph node involvement \\
\hline Stage 3: Tumor whose ablation is incomplete or impossible \\
\hline Stage 4: Presence of distant metastasis \\
\hline 1: The classification proposed by the Intergroup Rhabdomyosarcoma Study (IRS)
\end{tabular}

The treatment is based on surgery, multidrug therapy and radiotherapy.

The inguinal orchiectomy with high and early ligation of the spermatic cord represents the initial therapeutic time.

A hemiscrotectomy may be necessary in cases of locoregional invasion, history of trans-scrotal biopsy or oropexy [9-11,20]. The controversy is about the benefit of performing systematic retroperitoneal lymphadenopathy.

Currently it is considered that this removal is not necessary if the radiological investigations do not show any lymphonodal invasion, given the effectiveness of multidrug therapy in the control of micro metastases [7,9,11,20-22]. The appearance of a metastasis requires its removal if it is technically feasible [10,11].

In this case, multidrug therapy makes it possible to eradicate occult metastases [23]. It is indicated for all prognostic groups with a significant improvement in the probability of overall survival and progression-free survival. Several chemotherapy protocols have been used in the treatment of rhabdomyosarcomas. The associations IVE (ifosfamide, vincristine, etoposide) or IVA are the most used. The IRS IV test showed that the VAC protocol (vincristine, dactinomycin, cyclophosphamide) was as effective as the IVA and IVE protocols [4].

Radiation therapy is an important therapeutic arm in the management of rhabdomyosarcomas, it improves the local control rate and therefore the prognosis. It is indicated for groups II (microscopic residue) and III (macroscopic residue) and in case of lymph node involvement.

The late effects of radiation therapy are a limiting factor for children. However, technological advances have made it possible to improve irradiation techniques (conformal radiotherapy, with or without intensity modulation, and to spare adjacent risk organs [24].

Therapeutic indications depend on the pathological stage according to the IRS classification $[9,10,22,25]$ :

-Group I: orchiectomy followed by chemotherapy, no radiotherapy

-Groups II and III: this treatment is completed by retro-peritoneal lymphonodal dissection associated with local radiotherapy

-Group IV: orchiectomy then chemotherapy without lymphonodal dissection with excision of metastasis if it is technically feasible, supplemented by directed radiotherapy on retro-peritoneal lymphocytes and on metastases.

The prognosis for stage I and II rhabdomyosarcomas remains excellent with overall survival rates exceeding 90\% at 5 years [14,26].

The prognosis depends on several factors, the stage, the histological type and the response or not to the treatment. The embryonic form retains the best prognosis in its localized form. In addition, paratesticular rhabdomyosarcoma relapses are fatal.

The most common monitoring rhythm is every month during the first year, every two months during the following year, then every year for a period of five years, a term beyond which we can talk about healing. Nevertheless, we will not lose sight of the patient before 10 years of evolution.

Surveillance is based on clinical examination of both testicles and a complete examination searching other localization, and also a thoraco abdomino pelvic computed tomography (CT). The usefulness of the tumor marker assay is not specified in this pathology 
because of the small number of cases published in the literature and the frequent negativity of these as well as their lack of specificity.

\section{Conclusion}

Testicular rahbdomyosarcoma is a therapeutic and diagnostic emergency [14] but can be mistaken if the history of the patient is unclear as it can mimic a testicular torsion or infectious abnormalities. His care involves surgeons, radiologists, oncologist and anesthesists. The embryonic type is the most common (97\%) and has the best prognosis.

Several therapeutic pathways are being explored, including targeted therapies given the particular molecular profile of rhabdomyosarcomas. Adequate long-term surveillance should be instituted to detect relapses that are usually fatal.

\section{References}

1. Kabiri H, El Mansouri O, Albouzidi A (1996) Paratesticular rhabdomyosarcoma: a case report [Rhabdomyosarcome paratesticulaire: À propos d’un cas]. J Urol (Paris) 102: 176-9.

2. Abbou CC, Braun R, Chopin D (1991) Neoplasm of spermatic cord [Tumeur du cordon spermatique], Cancers congénitaux. Paris, Flammarion 381-8.

3. Grapin C, Boyer C, Gruner M (1994) Tumors of the testicle of the child [Les tumeurs du testicule de l'enfant]. J. Urol 100: 8-16.

4. Dasgupta R, Rodeberg DA (2012) Update on rhabdomyosarcoma. Semin Pediatr Surg 21: 68-78.

5. Le Guillou M, Perrin P (1981) Les tumeurs du testicule. [Rapport présenté au Congrès de l’AFU]. Paris, Masson.

6. Blyth B, Mandell J, Bauer SB, Colodny AH, Grierh E, et al. (1990) Paratesticular rhabdomyosarcoma : results of therapy in 18 cases. J Urol 144: 1450-3.

7. Ferrari A, Casanova M, Massimino M, Luksch R, Piva L, et al. (1998) The management of paratesticular rhabdomyosarcoma a single institutinal experience with 44 consecutive children. J Urol 159: 1031-4.

8. Gamba PG, Cecchetto G, Katende M, Carli M, Sotti G, et al. (1994) Paratesticular rhabdomyosarcoma (RMS) and paraaortic lymphadenectomy. Eur J Pediatr Surg 4: 158-60.

9. Asensio Lahoz LA, Abitua Bilbao J (1993) Paratesticular rhabdomyosarcoma. Diagnostic and therapeutic attitude [Rhabdomyosarcome paratesticulaire : attitude diagnostique et thérapeutique]. J Urol 99: 44-6.

10. Benchekroun A, Lachkar A, Soumana A, Farih MH, Belahnech Z, et al. (1998) Paratesticular rhabdomyosarcoma: about a case [Le rhabdomyosarcome paratesticulaire : à propos d'un cas]. Ann Urol 32: 107-10.

11. Kabiri H, El Mansari O, Al Bouzidi A, Taberkant M, Benomar S, et al. (1996) Rhabdomyosarcome paratesticulaire : à propos d'un cas. J Urol (Paris) 102: 176-9. 12. De Castro Barbosa F, Rosell Costa D, Aguera Fernández LG, Isa Kroon W, Sánchez de la Muela P, et al. (1991) Paratesticular rhabdomyosarcoma with intracaval neoplastic involvment : presentation of a case and review of the literature. Actas Urol Esp 15: 469-72.

13. Rodríguez-Rubio FI, Abad JI, Rábago G, Berián JM (1997) Long survival of a patient with paratesticular rhabdomyosarcoma with inferior vena cava involvement. Urology 50: 978-9.

14. Faure A, Diakité ML, Panait N, Chaumoître K, Rome A, Merrot T (2012) Le rhabdo- myosarcome para-testiculaire de l'enfant : une urgence scrotale. Arch Pediatr 19: 1340-4.

15. Dasgupta R, Rodeberg DA (2012) Update on rhabdomyosarcoma. Semin Pediatr Surg 21: 68-78.

16. Wu HY, Snyder HM 3rd, Womer RB (2009) Genitourinaryrhabdomyosarcoma:which treatment, how much, and when? J Pediatr Urol 5: 501-6.

17. Zheng L, Tang H, Chen X, Yang H, Yang M (2013) Paratesticular fetal-type rhabdo- myoma in a 12-year-old boy: a case report and literature review. Urology 82: $1150-2$.

18. Fikri M, Allali N, Dafiri R (2005) Une tumeur paratesticulaire rare :le rhabdomyosarcome. A propos de 8 cas. J Radiologie 86: 1565.

19. Ghorbal L, Abid W, Elloumia F, Sallemib T, Frikh M, et al. (2015) Embryonal paratesticular rhabdomyosarcoma: A case report and a literature revue [Rhabdomyosarcome embryonnaire paratesticulaire : à propos d'un cas et revue de la littérature]. Cancer Radiother 19: 334-6.

20. Benge BN, Byrd RL, Bergevin M, Winslow BH (1994) Scrotal recurrence of paratesticular rhabdomyosarcoma in a previously undescended testicle 5 years after orchiectomy. J Urol 152: 2117-8.

21. Gamba PG, Cecchetto G, Katende M, Carli M, Sotti G, et al. (1994) Paratesticular rhabdomyosarcoma (RMS) and paraaortic lymphadenectomy. Eur J Pediatr Surg 4: 158-60.

22. Hughes LL, Baruzzi MJ, Ribeiro RC, Ayers GD, Rao B, et al. (1994) Paratesticular rhabdomyosarcoma delayed effects of multimodality therapyand implications for current management. Cancer 73: 476-82.

23. Rodeberg D, Paidas C (2006) Childhood rhabdomyosarcoma. Semin Pediatr Surg 15: 57-62.

24. Terezakis SA, Wharam MD (2013) Radiotherapy for rhabdomyosarcoma: indicationsand outcome. Clin Oncol (R Coll Radiol) 25: 27-35.

25 Walsh PC, Retik AB, Vaughan ED, Wein AJ (1998) Pediatric oncology In: Campbell's Urology (7th Edn) Phi ladelphia, WB Saunders, USA.

26. Kurzrock EA, Busby JE, Gandour-Edwards R (2003) Paratesticular rhabdomyoma. J Pediatr Surg 38: 1546-7. 


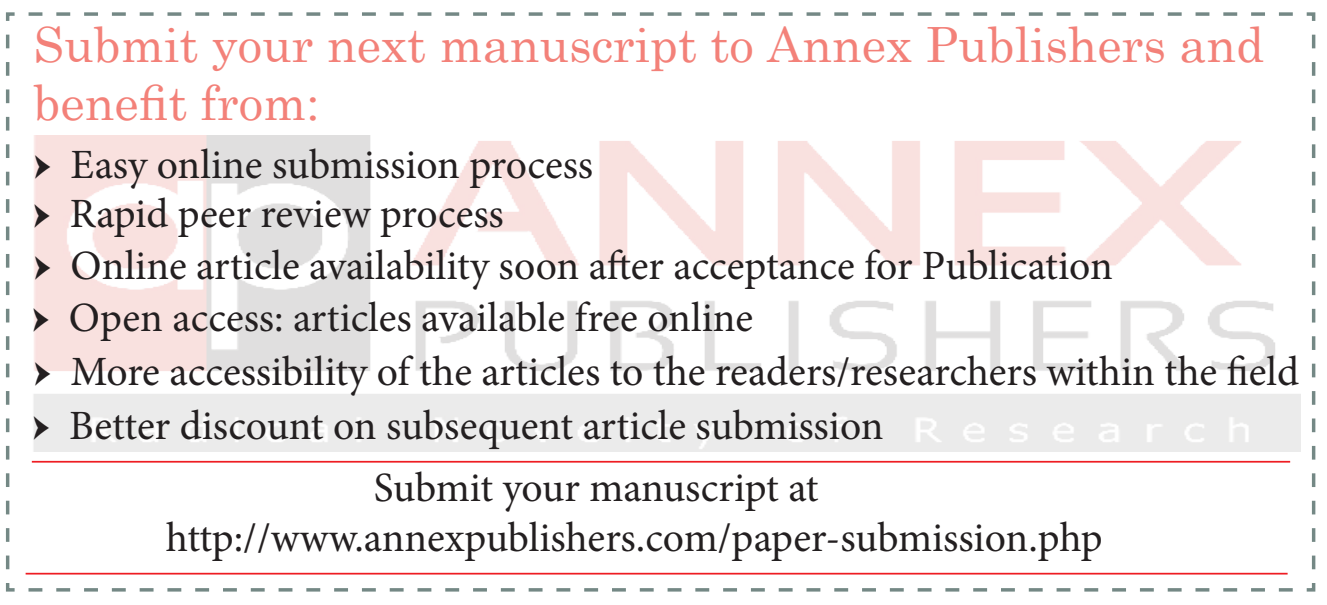

